\title{
Study of the characteristics of a sliding bearing under dynamic loading
}

\author{
Andrei Gorin ${ }^{1, *}$, Anton Sytin ${ }^{1}$, and Alexey Rodichev ${ }^{1}$ \\ ${ }^{1}$ Orel State University named after I.S.Turgenev, Komsomolskaya st. 95, 302026, Orel, Russia
}

\begin{abstract}
The article discusses the prerequisites and the process of creating an experimental bench for the study of external electromagnetic effects on rotor-bearing units in the work process. The control systems of the experimental setup and the electromagnetic device are presented, the latter is made in the form of 4 paired orthogonally located electromagnets. The percussion device can operate in a programmable mode. Flexible variation in speed and magnitude of the impact (according to a predetermined law of change) allows you to cover a wide range of possible external influences on the rotor unit. The tool for the automatic control of installation modules, as well as the collection and processing of experimental data, is software developed in the LabView visual programming environment. The drivers for matching the frequency converter and the controller, as well as all the software for the operation of the information-measuring system, the experiment and data processing, were written in the graphic programming language.
\end{abstract}

\section{Introduction and Problem formulation}

The problems of ensuring the vibrational reliability of rotor units are an urgent issue of today. The specified amplitude-frequency characteristics (AFC) and the operability of such systems are primarily associated with support units [1,2]. The use of elastically pliable plain bearings allows for reliable operation of the rotor assembly in a wide range of frequencies and loads, thanks to the adaptive bearing surface, necessary rigidity and high damping ability $[3,4]$. The priority direction for the development of rotor units is to increase productivity by increasing the operating speeds of rotation while minimizing weight and size characteristics. However, to ensure stable operation of the rotor, at high speeds, it is necessary to take into account a large number of parameters in the rotorsupport system, the determination of which allows to significantly optimize the design. In addition to the internal oscillations of the rotor in the bearings caused by an imbalance, the influence of a rotation drive, etc., situations of external impact on the rotor assembly body arise [5]. These situations, despite the probabilistic nature, can lead to a significant change in the trajectory of the rotor, which at significant speeds can lead to contact with the bearing surface, deformation and destruction of the rotor-bearing unit, with subsequent failure of the rotor assembly [6]. Therefore, experimental studies simulating dynamic effects are

\footnotetext{
* Corresponding author: gorin57@mail.ru
} 
relevant. In this case, it is necessary to control the dynamic effect to minimize time costs and obtain a complete picture in the studied object.

\section{Theoretical part}

The loads acting on the rotor can be divided into independent and dependent on the movement of the rotor. The first group includes loads that are constant in magnitude and direction (gravity, aerohydrodynamic forces, etc.), as well as various external forces (forces from maneuvering, foundation vibration, etc.). The second group consists of forces from imbalance, non-conservative aerohydrodynamic forces, reactions of supports and seals, forces of electromagnetic origin, etc. The dynamic behavior of the rotor under conditions of transient operation can be complex [7, 8]. The system "rotor - sliding bearings" together reacts to various kinematic and dynamic disturbances. A rotating shaft is a source of vibrations and at the same time an element that is subject to vibrations [9]. To study the influence of controlled dynamic effects on the rotor-bearing unit, a special experimental setup was taken as the basis (Fig. 1). In general, the experimental setup is a mechatronic system consisting of: a lobe gas-dynamic bearing, recording parts (displacement sensors), electromagnetic actuators and an electronic control system. The principle of operation of such a support is to register the deviation of the rotor from the equilibrium position by displacement sensors, the signal from which enters the electronic control system. After receiving the result of the deviation, the amplifier, powered by an external energy source, sends a signal to the electromagnets, which, acting on the rotor, set the necessary dynamic effect.



Fig. 1. Experimental setup.

1 - base, 2 - electric motor, 3 - support unit, 4 - electromagnetic unit, 5 - sensor node.

The experimental setup includes three main units:

- supporting unit (Fig. 2), consisting of a petal bearing with a deflection control system for elastic elements. However, any type of bearing with appropriate geometrical characteristics can be used.;

- electromagnetic unit (Fig. 3), necessary to set the external dynamic impact during operation;

- sensor unit that tracks rotor movements in two orthogonal directions, taking into account the skew of the rotor axis.

The support unit consists of two metal bearings with fixing elements and a seat for installing a petal bearing, which ensure its reliable mounting. Petal gas-dynamic bearings have a relatively simple design and principle of operation. The bearing surface of the bearing is formed by thin elastic metal plates. A low friction coefficient anti-friction coating is applied to the surface of the plates, which "lubricates" the bearing during start- 
up, until the shaft can rotate fast enough so that an air wedge forms between it and the elastic elements. As the rotor speed increases, the gas layer completely separates the working surfaces. The increase in speed is accompanied by an increase in the carrying capacity of the bearing.

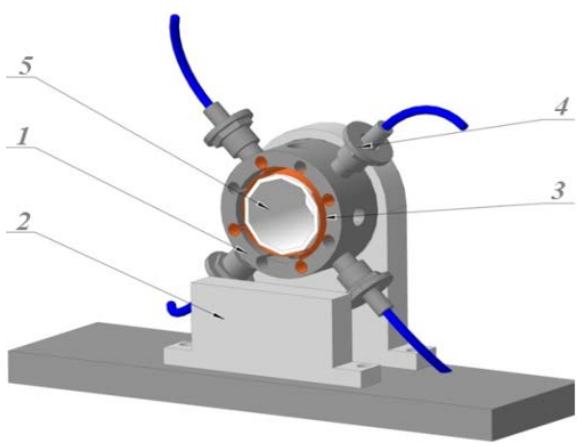

Fig. 2. General view of the support node.

1 - bearing, 2 - support stand, 3 - support stand, 4 - deflection sensor, 5 - rotor.

In the center of the experimental setup is an electromagnetic unit. Installation of the main parts is carried out on a bedplate. The supports have grooves for fixing the cores, on which in turn coils of electromagnets PME-211 are mounted.

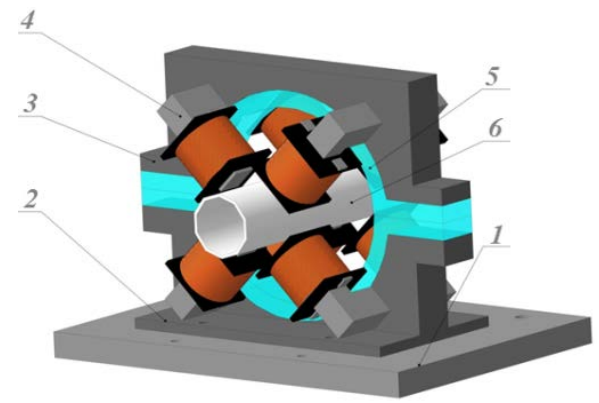

Fig. 3. Electromagnetic unit.

1 - base, 2 - bottom frame, 3 - upper frame, 4 - electromagnet, 5 - insulator, 6 - rotor.

An asynchronous electric motor is used as a rotor drive of the experimental setup rotor. The rotor of the experimental setup is a stepped shaft, at the end of which, with the help of a keyed connection, a hub with teeth is attached, which is part of the BoWex coupling.

The rotor mounted on the lobe gas-dynamic bearings with a dynamic electromagnetic loading device is a complex system with a large number of different control parameters (Figure 4). The ranges of variation of each of them are theoretically unlimited. Therefore, for automatic control of the experimental stand, as well as for the collection and processing of the obtained experimental data, an information-measuring system (IMS) was developed on the basis of the «National Instruments» software and hardware complex [10]. The basis of IMS is the multifunctional board NI6052E, which has the ability to multi-channel digital and analog I / O and counters-timers. The functional purpose of the board is to provide input-output signals, digitization and execution of commands, control of power executive modules of the stand. Signals from various sensors are fed into single or dual channel analog matching SCC modules.

Control of the motor and pump, as well as the emergency shutdown functions are performed via relay modules SC-RLY01. For a more compact arrangement and subsequent signal processing, the SCC and SC-RLY01 modules are mounted in a single SC-2345 block 
module, where the unified signals from the sensors are converted into the range perceived by the analog-to-digital converter $(0 . .5 \mathrm{~V})$.

In our particular case, displacement sensors and power modules of the experimental stand are connected to the SC-2345 through the interface connectors BNC, 9-Pin D-sub, Strain Relief. The SC-2345 module requires an external 5V power supply, the source of which is the SCC-PWR01 power supply. The NI6052E board and the SC-2345 module are commutated by a single SH 68-68-EP cable. Data from the NI6052E multifunction board, which has advanced synchronization and clocking capabilities, is sent to the controller for subsequent processing or recording to the hard disk.

\section{Experimental studies}

The experiment program includes at least three tests for the same operating parameters. Each test consists of accelerating the rotor to the operating speed, operating for some time at steady state, electromagnetic exposure and stopping. The capabilities of the experimental bench allow fixing the rotation frequency, trajectory of movement of the center of the axle of the rotor in the gap, the time-frequency characteristics of the rotor during acceleration and coasting, and other important parameters.

The development of an information-measuring system (IMS) for studying the dynamic characteristics of the rotor system was carried out taking into account the following basic requirements:

- simplicity of configuration and reconfiguration based on the unification of primary converters;

- simultaneous data collection and control of executive devices;

- the ability to simultaneously record data from all measurement channels;

- high metrological characteristics;

- displacement sensors must have a non-contact principle of operation, excluding the impact on a rotating rotor;

- have a convenient software package for outputting, processing and analyzing measurement results.

A photo of an experimental bench including a rotar unit and a controlled electromagnetic exposure device is shown in fig. 4 and fig.5.

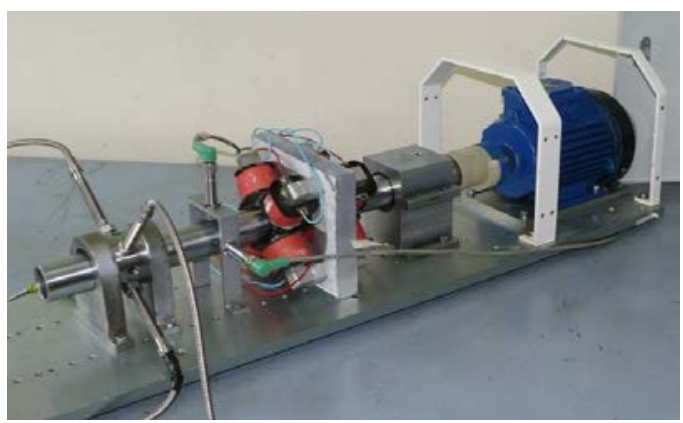

Fig. 4. Photo of an experimental bench with a controlled electromagnetic exposure system. 




Fig. 5. Photo of the experimental stand (location of displacement sensors).

The role of primary transducers is performed by eddy current transducers AR2100A-S05.05.1. The eddy current transducer consists of a non-contact eddy probe (primary transducer AE051.00.07), an extension cable (ARKZ950 / 3 connector) and an electronic unit (matching device D210A-C.05.05). The electronic unit generates a probe excitation signal and selects an informative parameter. The output signal is an electrical signal that is directly proportional to the distance from the end of the eddy probe to the controlled object. The transducer is often referred to as an eddy current sensor system. Two of these sensors are mounted in a frame in front of the bearing assembly and four in the bearing assembly (fig. 6) with their help, the position of the rotor in two mutually perpendicular planes and the deformation of the elastic elements are monitored.

A tool for automatic control of installation modules, as well as the collection and processing of experimental data, is software developed in the LabView visual programming environment. The drivers for matching the frequency converter and the controller, as well as all the software for the operation of the information-measuring system, the experiment and data processing, were written in the graphic programming language «G». The software for experimental research consists of two modules: 1) setting up and running an experiment; 2) processing experimental data. The module for tuning the informationmeasuring system and conducting the experiment in real time, integrated with the module for storing data and automated experiment, is used to visualize signals from all primary converters and their primary tuning. The developed program for conducting full-scale experiments includes an interface that displays settings for outputting results, a panel for generating a report, and sweep oscillations from displacement sensors.

As a result of a controlled experiment, the trajectories of the rotor motion in the ascent and steady-state motion mode were obtained (Fig. 6).

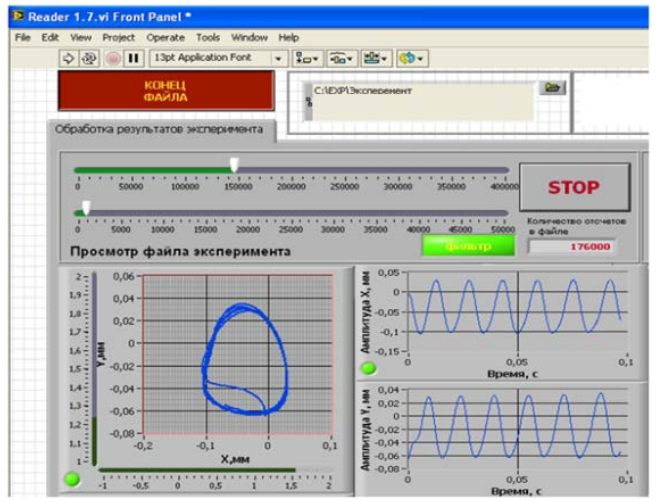

Fig. 6. Photo of the experimental stand (location of displacement sensors). 
Under the influence of controlled electromagnetic influence, the rotor trajectory in the plain bearings changes sharply (Fig. 7) and becomes chaotic in shape, depending on the direction and strength of the action, the geometrical parameters of the rotor-bearing assembly, as well as the kinematic motion parameters. There are two possible scenarios:

- loss of stability of motion, leading to an increase in the amplitude of oscillations, with subsequent collision of the rotor against the inner surface of the sliding bearing;

- vibration damping by the elastic forces of the lubricating layer of the sliding bearing, a gradual decrease in the amplitude of the oscillations and the return of the rotor to the stationary motion orbit.

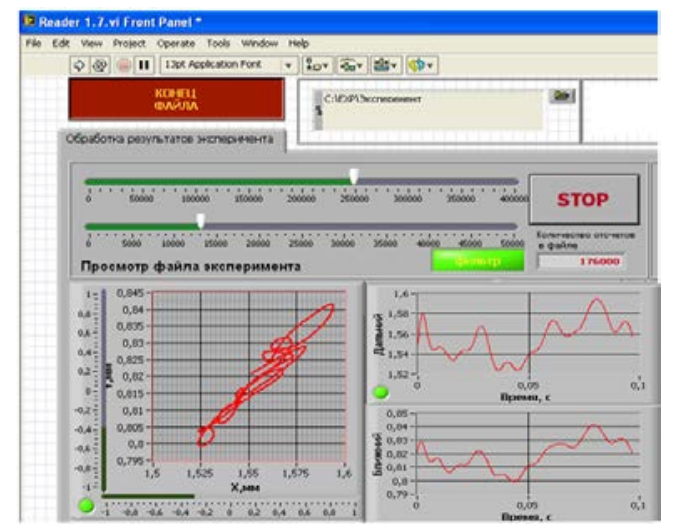

Fig. 7. The trajectory of the rotor under the action of external electromagnetic effects.

In the framework of this study, a separate software module was developed, which is intended for subsequent processing of stored experimental data. The Fourier analysis procedures built into the software are used as a filter for processing signals from sensors.

\section{Conclusions}

The experimental stand presented in the article with controlled electromagnetic action allows one to study the influence of external force factors on the trajectory and stability of the rotor motion in sliding bearings in manual and automatic mode, with the possibility of recording and subsequent analysis of the motion trajectories. Flexible variation in the speed and magnitude of the impact (according to a predetermined law of change) allows you to cover a wide range of possible external influences on the rotor unit.

\section{References}

1. O.V.Solomin, Method development for dynamic analysis of rotor systems with hydrodynamic bearings ( $\mathrm{PhD}$ dissertation, 2007)

2. E.L Poznyak, Rotors vibrations Machines, structures and their elements vibrations, Vibrations in machinery, v. 3, pp 130-189 (1980)

3. A.O. Pugachev, Transient modes dynamics of rotors with journal bearings ( $\mathrm{PhD}$ dissertation, 2004)

4. L.A. Savin, Theoretical basis of calculation and dynamics of sleeve bearings with liquid-vapor lubrication (PhD dissertation, 1998)

5. A.M. Aleksandrov, V.V. Filippov, Dynamics of Rotors (Moscow, MEI, 1995) 
6. V. A. Antipov, M.V. Duletskiy, R.N. Komarov, R.N. Polyakov, 2003 The influence of force factors on the characteristics of the "rotor-body" dynamic system, Mechanical engineering, Instrumentation,v. 3, pp. 6-9

7. V.A. Voskresenskiy, V.I. D'yakov, Calculation and design of sleeve bearings with liquid lubrication (Moscow, Mashinostroenie, 1980)

8. D.T. Gaevik, Bearing Supports in Modern Machinery (Moscow, Mashinostroenie, 1985)

9. A.G. Kostyuk, Dynamics and Durability of Turbomachinery (Moscow, MEI, 2000)

10. A.V. Barkov, N.A. Barkova, A.Y. Azovtsev, Vibration Monitoring and Diagnostics of Rotary Machines (SPbGMTU, 2000) 